\title{
Acoustic-Emission Study of Intermittency of Plastic Flow during Twinning and Dislocation Glide
}

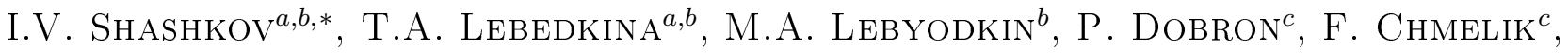

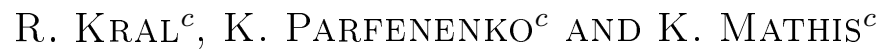 \\ ${ }^{a}$ Institute of Solid State Physics, Russian Ac. Sci., 142432 Chernogolovka, Russia \\ ${ }^{b}$ Université de Lorraine, LEM3, UMR CNRS 7239, Ile du Saulcy, 57045 Metz, France \\ ${ }^{c}$ Department of Physics of Materials, Charles University, Ke Karlovu 5, CZ-121 16 Prague 2, Czech Republic
}

\begin{abstract}
Recent studies of plastic deformation with the aid of acoustic emission techniques proved an intermittent, scale-invariant character of plastic processes, as reflected in power-law statistical distributions. In some cases, the power-law exponents display close values leading to hypothesis of universality of scaling laws for various mechanisms of plasticity. Nevertheless, the accurate determination of the power law may be impeded by some sources of errors inevitable in real conditions, in particular, by superposition of individual acoustic emission events. In the present work, the sensitivity of the apparent statistics to the variation of the parameters of individualization of acoustic emission events is examined using $\mathrm{Mg}$ and $\mathrm{Al}$ based alloys. Both these alloys exhibit a highly cooperative character of plastic deformation, leading to strong acoustic activity which is governed by distinct microscopic mechanisms - mechanical twinning and the Portevin-Le Chatelier effect, respectively.
\end{abstract}

PACS: 62.20.F-, 62.65.+k, 05.65.+b, 64.60.av

\section{Introduction}

Recent studies of plastic deformation using high-resolution experimental techniques, in particular the acoustic emission (AE), proved an avalanche-like character of plastic processes, which is reflected in critical-type power-law statistical distributions of the measured variables. The power-law statistics was found for different mechanisms of plasticity: during macroscopically smooth plastic flow of pure materials controlled by the dislocation glide [1, 2], under conditions of macroscopic plastic instability caused by interaction of dislocations with solute atoms - the Portevin-Le Chatelier (PLC) effect [3], or during mechanical twinning [1]. These observations imply an intrinsically intermittent and scale-invariant mechanism of plasticity on a "mesoscopic" scale which is associated with the collective motion of crystal defects and manifests itself through the concomitant AE. Moreover, similar values of the power-law exponents found for dislocation glide and twinning in single crystals of various materials, including ice and a number of metals, have led to a hypothesis of universality of the corresponding scaling laws. At the same time, the exponents determined for polycrystalline ice differ from this unique value [4]. Considerably different power-law indices are found for the PLC effect [3]. These observations bring up a question of reliability of such data. Indeed, the apparent values of the critical exponents may differ from their true values for several reasons [5, 6]. Particularly, the avalanches may either closely follow each other or

* corresponding author; e-mail: ivan.shashkov@univ-lorrain.fr occur almost simultaneously in different locations in the sample, and be recorded as a single event. It is not clear a priori how such overlap of individual events would affect the results of the statistical analysis. The other sources of errors stem from insufficient resolution of the individual events against noise, acoustic echo, and so on. All these factors are affected by the criteria utilized to identify the events within the acoustic signal. Up to now, the sensitivity of the apparent statistics to these criteria has not been verified experimentally, although the problem is general and concerns a vast range of dynamical systems of different nature which are characterized by depinning transitions and avalanche-like behavior [5].

In the present paper, the effect of the parameters of individualization of acoustic events on their statistics is examined using various materials the plastic deformation of which is accompanied by strong acoustic activity and controlled by different microscopic mechanisms: a combination of twinning and dislocation glide in hexagonal $\mathrm{Mg}$ alloys and the PLC effect in face-centered cubic $\mathrm{AlMg}$ alloys.

\section{Experimental technique and data processing}

Several materials were examined in the tests: (i) $\mathrm{MgZr}$ alloys with different $\mathrm{Zr}$ content which influenced on the grain size (material with $0.04 \mathrm{wt} \%, 0.15 \mathrm{wt} \%$, and $0.35 \mathrm{wt} \%$ of $\mathrm{Zr}$ had the average grain size of $550 \mu \mathrm{m}$, $360 \mu \mathrm{m}$, and $170 \mu \mathrm{m}$, respectively); (ii) magnesium alloy AZ31 (2.9 wt\% Al, 0.98 wt\% Zn, 0.29 wt\% Mn) subjected to extrusion at different temperature, $100^{\circ} \mathrm{C}$ or $30{ }^{\circ} \mathrm{C}$, in order to obtain fine-grained (about $3 \mu \mathrm{m}$ ) or coarser-grained $(16 \mu \mathrm{m})$ polycrystalline structure; (iii) cold-rolled $\mathrm{AlMg}$ alloys with $3 \%$ or $5 \%$ of $\mathrm{Mg}$, which 
were either tested as-rolled or preliminarily subjected to solution annealing $\left(400^{\circ} \mathrm{C}, 2 \mathrm{~h}\right)$ followed by water quenching. All tests were carried out at room temperature. Magnesium alloys were deformed both by tension (AZ31 samples in the form of cylinders $35 \mathrm{~mm}$ long and $6 \mathrm{~mm}$ in diameter; $\mathrm{MgZr}$ samples in the form of square boxes $5 \times 5 \times 25 \mathrm{~mm}^{3}$ ) and compression (AZ31 cylinders $16.5 \mathrm{~mm}$ long and $11 \mathrm{~mm}$ in diameter) with a constant nominal strain rate $\dot{\varepsilon}_{\mathrm{a}}$ in the range from $1 \times 10^{-4} \mathrm{~s}^{-1}$ to $1 \times 10^{-3} \mathrm{~s}^{-1}$. Since the PLC effect displays various types of deformation curves which reflect the occurrence of various spatiotemporal strain patterns depending on $\dot{\varepsilon}_{\mathrm{a}}$ [7], AlMg alloy (flat specimens typically $30 \mathrm{~mm}$ long and $7 \mathrm{~mm}$ wide) was tested by tension in a wide $\dot{\varepsilon}_{\mathrm{a}}$-range from $2 \times 10^{-5} \mathrm{~s}^{-1}$ to $6 \times 10^{-3} \mathrm{~s}^{-1}$.

The acoustic emission technique primarily used in the present study was based on the DAKEL-CONTI-4 system which allows continuous sampling (with the frequency of $2 \mathrm{MHz}$ ) of the $\mathrm{AE}$ signal arriving from a piezoelectric transducer with a frequency band of 100$600 \mathrm{kHz}$ [8]. This makes possible a comprehensive post-processing of the complete stored signal using various parameters of individualization of the AE events. Besides, the equipment allows for the standard procedure of picking out acoustic events ("hits") using preset parameters, i.e., without data streaming. Namely, the software continuously detects the crossings of a threshold voltage $U_{0}$ and the local maxima of the signal. An event is considered to start when the acoustic signal surpasses $U_{0}$. The current maximum is recorded as the event's peak amplitude $A$, provided it was not exceeded during the so-called peak definition time (PDT). The end of the event is detected when the signal remains below $U_{0}$ for a period exceeding a hit definition time (HDT). Afterwards, no measurement is performed during a hit lockout time (HLT) in order to filter out sound reflections. Obviously, the choice of $U_{0}, \mathrm{HDT}$, and HLT may influence on the identification of the AE events. The conventional AE studies apply the following rules of thumb to set the time parameters. In one approach, a high value is chosen for HDT in order to include all sound reflections into the event. HLT can then be taken small. The disadvantage of this approach is that the event duration and the related parameters, such as the AE energy, are loosely determined. In the opposite case, a small HDT is set in order to separate the hit from the sound reflections which are then cut off by choosing a large HLT. This method is not free from drawbacks either, because it results in a lost of a part of the useful signal, particularly, the "aftershocks" that may follow the initial plastic event [1]. In any case, the criteria of "smallness" or "largeness" are approximate.

The events identification in a continuously stored AE signal mimicked this standard procedure in order to ensure the conformity of the results obtained by different methods and for different datasets. Each of the three parameters, $U_{0}$, HDT, and HLT, was varied in a wide range of magnitude while keeping the other parameters constant.
The sensitivity of the data stream measurements occurred to be insufficient for careful analysis of the data obtained for $\mathrm{AlMg}$ samples. In this case, a Physical Acoustics LOCAN 320 system was utilized, which did not provide data stream but recorded series of acoustic events using preset parameters. In these tests, $U_{0}$ was set at $27 \mathrm{~dB}$ - the value corresponding to the noise level for the free-running deforming machine - and the effect of changing the HDT and HLT by a factor of ten was tested at different strain rates.

Usually, the peak amplitude $A$ of the acoustic events, which is believed to reflect the magnitude of the corresponding strain-rate bursts, is studied by the statistical analysis [1-3]. In Ref. [9], it was argued that the distributions of the squared amplitude $A^{2}$ reflect the statistics of the energy dissipated by viscoplastic deformation during an event. This approach was adopted in subsequent works (e.g., [3]) and will be used in what follows.

\section{Experimental results and discussion}

During the last decade, the AE accompanying the plastic deformation of $\mathrm{Mg}$ and $\mathrm{Al}$ alloys was studied in detail. It has been shown to depend on the microscopic mechanisms of plasticity, the type of loading, the specimen geometry, the deformation stage, the strain rate, and temperature (e.g., $[10,11])$. In spite of this diversity, the investigation performed in the present study revealed a persistent power-law character of the amplitude statistics of AE. Such robustness allowed analyzing the sensitivity of the experimental estimates to the event identification criteria. A detailed analysis of the statistics of the amplitude and other characteristics of $\mathrm{AE}$ in relation with the relevant deformation processes goes beyond the scope of the present paper and will be published elsewhere. Below, the effect of the above-discussed parameters on the power-law exponents is analyzed using the examples of $\mathrm{MgZr}$ and $\mathrm{AlMg}$ alloys. These materials are chosen as representing two distinct cases. Indeed, it is known that both the wave forms of the individual acoustic events and the overall AE behavior have different signatures in the case of twinning or dislocation glide $[1,12]$.

For all samples, and similarly to other materials, high nonstationary AE activity is observed at small strains, in the region of the elastoplastic transition. It further decreases and displays roughly stationary behavior, which allows performing the statistical analysis in a steady-state range. Figure 1 presents an example of an acoustic signal recorded during tension of a specimen of the $\mathrm{Mg} 0.35 \% \mathrm{Zr}$ alloy for which the AE is known to be essentially governed by twinning [11].

The conformity of the statistics for the events which are either extracted from the continuously recorded signal or detected by the equipment using preset parameters was first verified as illustrated in Fig. 2. In this test, the threshold $U_{0}$ was chosen to be equal to $16.48 \mathrm{mV}$ in the former case of the continuously measured signal, which corresponds to the logarithmic threshold of $45 \mathrm{~dB}$ 


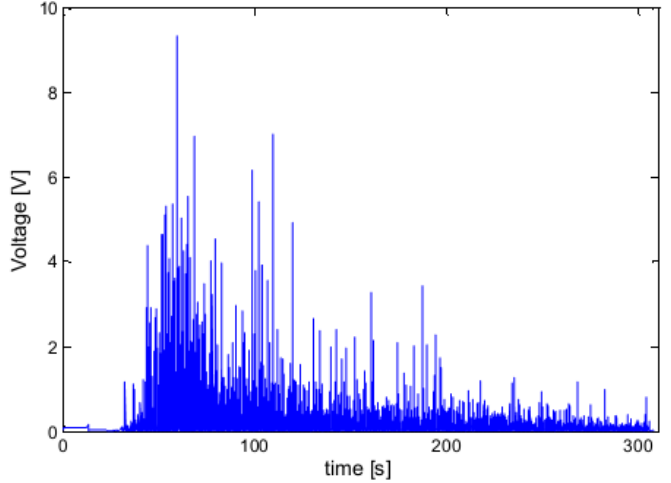

Fig. 1. Example of a series of AE recorded during tension of a Mg0.35 wt\%Zr sample at $\dot{\varepsilon}_{\mathrm{a}}=3.5 \times 10^{-4} \mathrm{~s}^{-1}$. Intervals of stationary behavior where the statistical analysis was performed were selected for $t>120 \mathrm{~s}$.

in the latter case. The time parameters were set at $\mathrm{HDT}=800 \mu \mathrm{s}$ and HLT $=100 \mu \mathrm{s}$. Despite the discreteness of the logarithmic measure allowed by the standard procedure, the series of events selected with the aid of two different methods coincide with high accuracy. It can be seen in Fig. 2 that both methods result in power-law dependences over more than three orders of magnitude of $A^{2}$ and give close values for the exponent $\alpha$ determined as a least squares estimate of the slope of the dependences in double-logarithmic coordinates: $\alpha=-1.80 \pm 0.03$ and $\alpha=-1.78 \pm 0.03$ for the "continuous" and "discrete" methods, respectively.

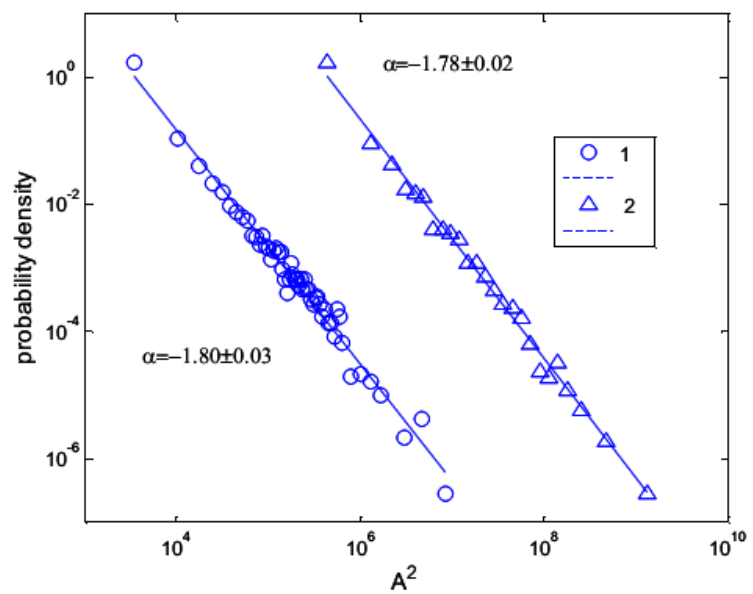

Fig. 2. Comparison of statistical distributions for the $\mathrm{AE}$ events either extracted from the continuously recorded signal (1) or detected during the test using preset parameters (2). Data for the same sample as in Fig. 1.

Figure 3 shows examples of the probability functions illustrating the effect of $U_{0}$ for one particular choice of time parameters, HDT $=50 \mu \mathrm{s}$ and HLT $=100 \mu \mathrm{s}$. It can be recognized that the statistics obeys power laws in a wide $U_{0}$-range and the corresponding slopes are fairly robust. The main effect of the increase in $U_{0}$ consists in the reduction of the number of events and the corresponding limitation of the interval of $A^{2}$ because of the cutoff of the low-amplitude events. Nevertheless, some decrease in $\alpha$ can be also detected: $\alpha=-1.80 \pm 0.05$ for $U_{0}=9 \mathrm{mV}$ and $\alpha=-1.87 \pm 0.02$ for $U_{0}=60.1 \mathrm{mV}$.

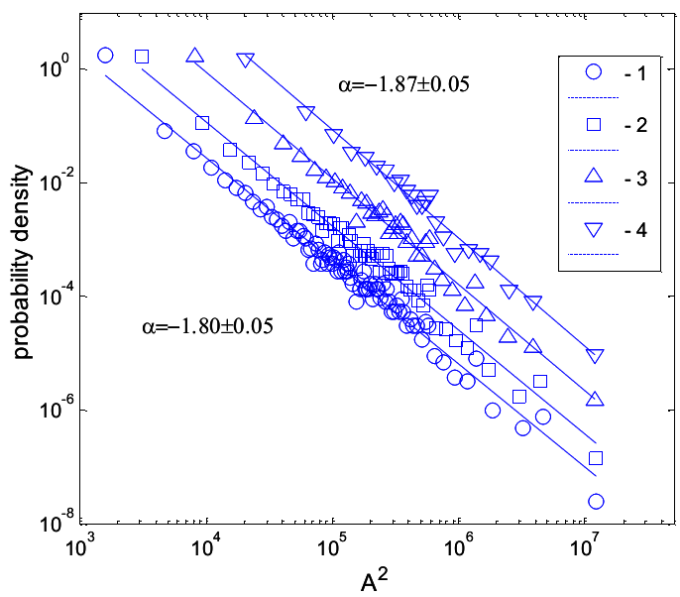

Fig. 3. Effect of the voltage threshold on the statistics of the amplitudes of $\mathrm{AE}$ events for the same specimen. $1-U_{0}=9 \mathrm{mV}, 2-U_{0}=15.3 \mathrm{mV}, 3-U_{0}=30.5 \mathrm{mV}$, $4-U_{0}=60.1 \mathrm{mV}$.

Using families of such curves, the dependences of $\alpha$ on $U_{0}$, HDT, and HLT were traced in large ranges of variation of each parameter, as illustrated in Fig. 4. Figure 4a represents $\alpha\left(U_{0}\right)$-curves for three $\mathrm{MgZr}$ alloys and for two choices of the time parameters. It can be recognized that $\alpha$ relatively strongly depends on $U_{0}$ only in a narrow range $U_{0}<10 \mathrm{mV}$ (even here, all changes in $\alpha$ do not exceed 0.2 ). The rate of the dependence falls with increasing $U_{0}$. A weak or no dependence (within the error bars) is observed for $U_{0}$ in the range from $10 \mathrm{mV}$ to the maximum value of $90 \mathrm{mV}$, above which the amount of data becomes too small for the statistical analysis. Most probably, the initially lower absolute $\alpha$-value is explained by the merging of successive $\mathrm{AE}$ events when $U_{0}$ is low, so that the number of smaller events is decreased and the apparent power law is flatter than the true one. Indeed, such merging can take place for low $U_{0}$ values if the individual $\mathrm{AE}$ hits are linked to each other due to the presence of a continuous background, e.g., noise. This suggestion is consistent with the changes observed when HDT is reduced from $800 \mu$ s to $50 \mu$ s: since less effective overlapping of successive hits is expected for the lower HDT, the initial fall in the dependence becomes sharper and $\alpha$ quickly saturates at an approximately constant value. The weak dependences which can be detected in the almost saturated region may also be due to the influence of the statistics depletion. In any case, an important conclusion following from this figure is that the effect observed is weak in a wide $U_{0}$-range. Furthermore, for each 

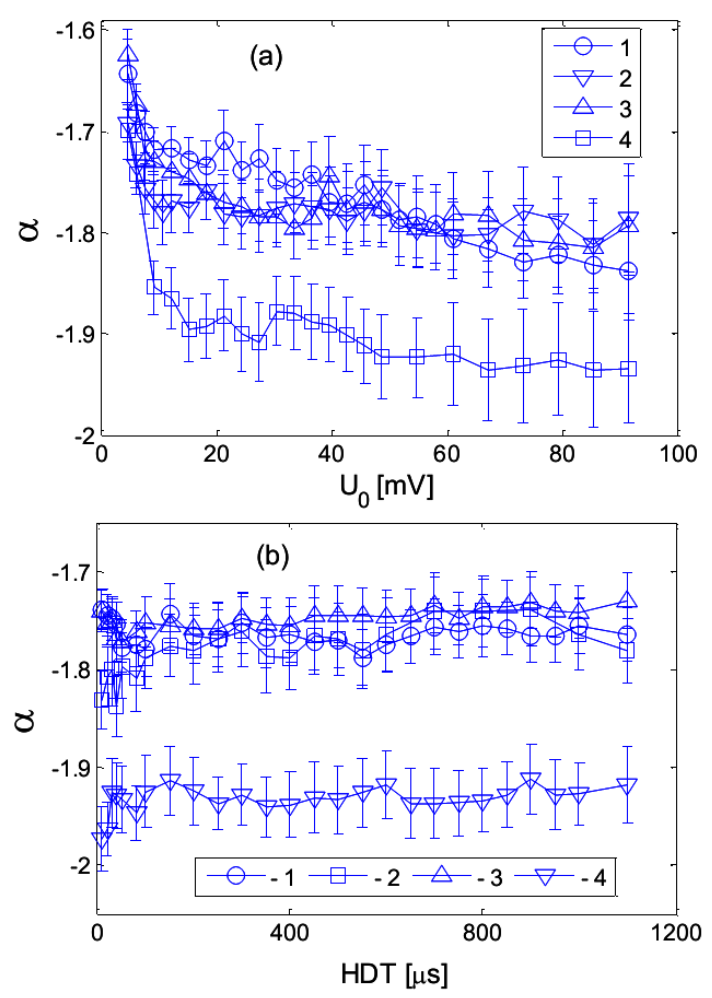

Fig. 4. Effect of (a) $U_{0}$ and (b) HDT settings on the power-law index $\alpha$ for MgZr specimens deformed at $\dot{\varepsilon}_{\mathrm{a}}=$ $3.5 \times 10^{-4} \mathrm{~s}^{-1} .1$ and $2-\mathrm{Mg} 0.35 \% \mathrm{Zr}, 3-\mathrm{Mg} 0.15 \% \mathrm{Zr}$, $4-\mathrm{Mg} 0.04 \%$ Zr. (a) HLT $=100 \mu \mathrm{s}$; HDT $=800 \mu \mathrm{s}$ except for the case (2) where HDT $=50 \mu \mathrm{s}$. (b) HLT $=$ $0 \mu \mathrm{s} ; U_{0}=17 \mathrm{mV}$, except for the case $(2)$ where $U_{0}=$ $67 \mathrm{mV}$.

HDT, a threshold value can be found above where its influence is insignificant.

Another interesting observation is that whereas two of the three kinds of samples display close $\alpha$-values, the curve obtained for the material with the largest grain size goes separately. In spite of this quantitative difference, all the curves have the same shape described above. Thus, although the different power law indicates a different specific structure of the AE signal in $\mathrm{Mg} 0.04 \% \mathrm{Zr}$, a similar effect of $U_{0}$ is found. Such robustness testifies that the quantitative difference between the materials with different microstructure is not due to artifacts of the AE method but reflects physically sound changes in the correlation of the deformation processes (see, e.g., [11]).

The companion Fig. $4 \mathrm{~b}$ represents the effect of HDT for the same tests and for two $U_{0}$-levels: one selected just above the range of the sharp $\alpha\left(U_{0}\right)$-dependence $(17 \mathrm{mV})$ and another taken in the far saturation region $(67 \mathrm{mV})$. It can be recognized that the effect of HDT is weak in both cases and almost negligible for $U_{0}=17 \mathrm{mV}$. Some variation of $\alpha$ in a narrow range of small HDT values can be detected for $U_{0}=67 \mathrm{mV}$ and is most likely due to the statistics depletion. As a whole, the analysis is quite robust against HDT variation. As far as the HLT

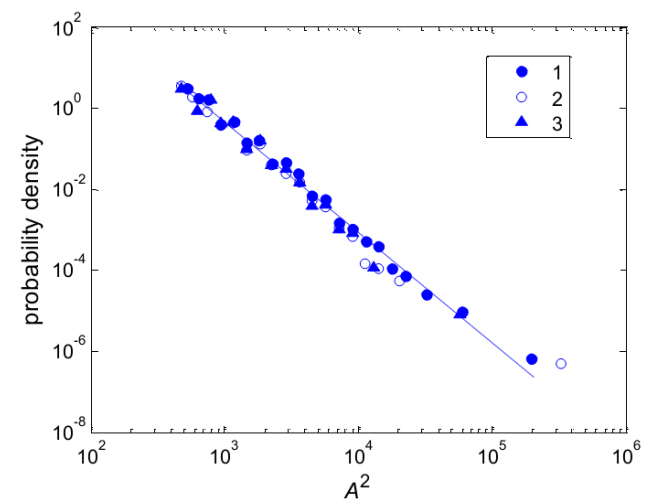

Fig. 5. Probability density function for squared amplitude of AE events collected in the same strain range for three different samples deformed at driving strain rate $\dot{\varepsilon}_{\mathrm{a}}=2 \times 10^{-5} \mathrm{~s}^{-1} .1$ and $2-\mathrm{HDT}=\mathrm{HLT}=300 \mu \mathrm{s} ;$ $3-\mathrm{HDT}=\mathrm{HLT}=40 \mu \mathrm{s}$.

is concerned, no significant dependence was found over the whole range studied, from 0 to $1 \mathrm{~ms}$.

Finally, Fig. 5 presents examples of AE amplitude statistics for three AlMg samples. All samples were deformed in the same experimental conditions but the time settings used to detect the AE events were different. The analyzed data are normalized with regard to the average over the respective dataset and all three dependences fall onto one master curve, probably except for some deviations from the power law for the largest events. This and similar results obtained for different $\dot{\varepsilon}_{\mathrm{a}}$ testify that the power law observed for AlMg alloys is also robust against the variation of HDT and HLT.

\section{Conclusions}

Power-law statistics is found for the amplitude of AE events accompanying plastic flow of various materials in different experimental conditions. The data obtained confirm the hypothesis that the plastic deformation is inherently intermittent, critical-type process at the scale relevant to $\mathrm{AE} \mathrm{[1-4].} \mathrm{The} \mathrm{major} \mathrm{result} \mathrm{of} \mathrm{the} \mathrm{present}$ study is that the criteria used to identify the individual AE events weakly influence on the apparent AE statistics. This conclusion has been verified using $\mathrm{Mg}$ and $\mathrm{Al}$ alloys which are characterized by distinct deformation mechanisms and display different AE behaviors. Not only this robustness justifies the quantitative estimates of the critical indices, but also it provides an additional proof of the above hypothesis. Indeed, one of the consequences of the variation of the event identification parameters is the cutoff of a part of data from the entire statistical sample. The robustness of the statistics against such cutoff is consistent with the scale invariance reflected in the power-law dependences.

More specifically, almost no effect was discerned when HDT and HLT were varied. Somewhat stronger, albeit a weak effect is observed in a wide range of the voltage threshold $U_{0}$. The only case when a relatively strong 
influence on the power-law is found corresponds to a narrow range of the lowest $U_{0}$ values and is most likely due to aggregation of successive $\mathrm{AE}$ events.

\section{Acknowledgments}

This work was supported by the Research Goal MSM0021620834 that is financed by the Ministry of Education, Youth and Sports of the Czech Republic and from the research grant P108/11/1267 financed by the Czech Science Foundation. The work received also support from the French Ministry of Foreign Affairs, and the Ministry of Education, Youth and Sports of the Czech Republic within the framework of the mobility program Contact-Barrande MEB 021004 (21971TH). K.P. is also grateful for financial support from the Grant Agency of Charles University No. 166310 and from the grant SVV-2011-263303.

\section{References}

[1] T. Richeton, P. Dobron, F. Chmelik, J. Weiss, F. Louchet, Mater. Sci. Eng. A 424, 190 (2006).

[2] J. Weiss, T. Richeton, F. Louchet, F. Chmelik, P. Dobron, D. Entemeyer, M. Lebyodkin, T. Lebedkina, C. Fressengeas, R.J. McDonald, Phys. Rev. B 76, 224110 (2007).
[3] Y. Bougherira, D. Entemeyer, C. Fressengeas, N.P. Kobelev, T.A. Lebedkina, M.A. Lebyodkin, J. Phys. Conf. Series 240, 012009 (2010).

[4] T. Richeton, J. Weiss, F. Louchet, Nature Mater. 4, 465 (2005).

[5] R.A. White, K.A. Dahmen, Phys. Rev. Lett. 91, 085702 (2003).

[6] C. Fressengeas, A.J. Beaudoin, D. Entemeyer, T. Lebedkina, M. Lebyodkin, V. Taupin, Phys. Rev. B 79, 014108 (2009).

[7] P. Rodriguez, S. Venkadesan, Solid State Phenom. 42-43, 257 (1995).

[8] R. Král, P. Dobroň, F. Chmelík, V. Koula, M. Rydlo, M. Janeček, Kovove Mater. 45, 159 (2007).

[9] J. Weiss, J.R. Grasso, M.C. Miguel, A. Vespignani, S. Zapperi, Mater. Sci. Eng. A 309-310, 360 (2001).

[10] F. Chmelík, A. Ziegenbein, H. Neuhäuser, P. Lukáč Mater. Sci. Eng. A 324, 200 (2002).

[11] K. Máthis, J. Čapek, Z. Zdražilová, Z. Trojanová, Mater. Sci. Eng. A 528, 5904 (2011).

[12] A. Vinogradov, D.L. Merson, V. Patlan, S. Hashimoto, Mater. Sci. Eng. A 341, 57 (2003). 